\title{
Isolation and identification of soil bacteria of the genus Bacillus
}

Kalinin A.V.*, Tsygankova O.I., Koteneva E.A., Abramovich A.V.

Stavropol Antiplague Institute of the Rospotrebnadzor, Stavropol, Russia

*e-mail: jugask@mail.ru

Key words: soil bacilli, isolation, identification, mass spectrometry

Motivation and Aim: Necessity to carry out indication of Bacillus anthracis not only in biomaterial, but also in environmental objects containing a great number of bacteria of this genus requires testing the specificity of applied methods, test systems and diagnostic preparations on a representative collection of strains of closely related saprophytes.

The aim of the work was to isolate soil representatives of the genus Bacillus, to carry out their identification and to store them in the form of a working collection for experimental and production purposes.

Methods and Algorithms: We studied 68 samples of soil from steppe areas, pastures, settlements, cattle-breeding farms of various areas of Stavropol Territory and neighboring republics. Liquid suspensions of soil were inoculated on Crome ${ }^{\mathrm{TM}}$ Bacillus Agar (HIMEDIA) and twenty-four hours later colonies differing from each other in their morphology and in the character of their change of medium were selected from the surface of the medium and subcultured on LB agar. Samples were prepared by lysis of 18-hour vegetative cultures in $80 \%$ TFU with subsequent ultramicrocentrifuge filtration. Identification of cultures it was carried out by the method of MALDI TOF MS using the device Microflex LRF Bruker. For identification we used the commercial database of Bruker company (version Bruker Taxonomy V 7). Spores were grown on KG Agar (HIMEDIA) and stored in cryoprotector (Deltalab) at $-20^{\circ} \mathrm{C}$.

Results: 243 cultures of 20 species of the genus Bacillus have been isolated and identified: B. antrophaeus, B. asahii, B. cereus, B. firmus, B. halotolerans, B. horneckiae, $B$. idriensis, B. licheniformis, B. marisflavi, B. megaterium, B. pseudomycoides, $B$. pumilis, B. siamensis, B. simplex, Bacillus sp. (cluster of strains of B.cereus group the species of which were not identified), B. sporothermodurans, B. subtilis, Lysinobacillus boronitolerans, Lysinobacillus fusiformis, Lysinobacillus sphaericus, Viridibacillus neidei.

Conclusion: The isolated cultures may be used for testing the specificity of various test systems and preparations for the diagnosis of anthrax, and the database of mass spectrafor specific identification of representatives of the genus Bacillus.

\section{References}

1. Vilain S., Luo Y., Hildreth M.B., Brözel V.S. Analysis of the life cycle of the soil saprophyte Bacillus cereus in liquid soil extract and in soil. Applied Environmental Microbiol. 2006.

2. Rahi P., Prakash O., Shouche Y.S. Matrix-assisted laser desorption/ionization time-of-flight massspectrometry (MALDI-TOF MS) based microbial identifications: challenges and scopes for microbial ecologists front. Microbiol. 2016. 